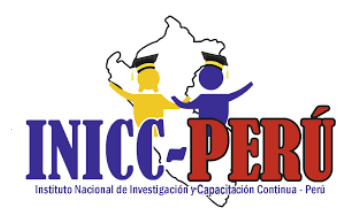

\title{
La realidad virtual en la experiencia educativa de pregrado
}

Virtual reality in the undergraduate educational experience

\author{
CYNTHIA MELISSA ELIAS RAMOS ${ }^{1}$
}

https://orcid.org/0000-0002-9066-6257

\section{SERGIO IVAN VARGAS APARCANA ${ }^{2}$}

https://orcid.org/0000-0002-3100-1941

\section{KARLA VANESSA BEATRIZ CASTILLO LY ${ }^{3}$}

https://orcid.org/0000-0002-7448-7486

\begin{abstract}
RESUMEN: Frecuentemente los alumnos de pregrado tienen dificultades para lograr propuestas y resolver problemas en forma creativas, lo cual tiene como consecuencia el bajo rendimiento en algunas asignaturas universitarias. El objetivo de este artículo es analizar cómo la realidad virtual (RV) mejora la experiencia educativa de pregrado, a través del análisis de artículos referentes al tema de los últimos tres años. Se tiene como conclusión general que la realidad virtual mejora la creatividad a través de la motivación y el estado de flujo, desarrollando habilidades para el aprendizaje en los estudiantes de pregrado, fomentando su uso y la adaptación de los programas existentes a las clases para que los alumnos generen más soluciones creativas ante los retos y problemas existentes.
\end{abstract}

Palabras clave: realidad virtual; educación; creatividad; motivación; estado de flujo.

ABSTRACT: Undergraduate students often have difficulties coming up with proposals and solving problems creatively, which results in poor performance in some university subjects. The objective of this article is to analyze how Virtual Reality (VR) improves the undergraduate educational experience, through the analysis of articles referring to the subject from the last three years. The general conclusion is that virtual reality improves creativity through motivation and the state of flow, developing skills for learning in undergraduate students, promoting its use and the adaptation of existing programs to classes so that students generate more creative solutions to existing challenges and problems.

Keywords: Virtual Reality; Education; Creativity; Motivation; Flow State. 


\section{INTRODUCCIÓN}

La realidad virtual (RV), es un campo tecnológico que crea nuevos entornos o copia los ya existentes. Para ello se utiliza un ordenador y se visualizan de manera inmersiva a través de unas gafas de realidad virtual, teniendo una visión 3D y $360^{\circ}$, pudiendo llegar a sentir que nos encontramos en ese lugar (Gómez Sánchez, 2018). Siendo su uso mediante cascos de Oculus Rift, Daydream, Play Station VR o VR Sky, además del empleo de celulares con unas gafas, que se pueden realizar manualmente de cartón facilitando su consumo. (Gutiérrez et al., 2019). Así tenemos la capacidad de construir e interactuar con los objetos 3D; realizar representaciones en forma de avatar; y comunicarse con otros participantes (Grigorenko, 2019).

De esta manera, la RV tiene el potencial de mejorar el aprendizaje de los estudiantes brindando experiencias en primera persona, como la posibilidad de la participación del alumno, junto con la capacidad de explorar, manipular y observar: objetos, estructuras, representaciones y entornos virtuales. Por lo que promueven aprendizajes más profundos y constructivistas, que cuando simplemente se escucha o se ve (Gutiérrez et al., 2019). También, el conocimiento virtual dado, puede transmitir el desarrollo de habilidades y destrezas que se necesiten para actuar en situaciones de la realidad (Kampling, 2018). En tal sentido, el uso la RV en la educación, contribuye a motivar y estimular en forma virtual a los alumnos para que se involucren totalmente en el proceso, interactuando con la RV y observando los resultados que posteriormente integrar y retener información, además de desarrollar habilidades de aprendizaje, como la creatividad (Tomalá Suárez \& Obando Chonillo, 2018).

Actualmente se está usando la RV en la educación universitaria, logrando la inmersión en situaciones variables y complejas, por medio de las simulaciones, para realizar recorridos virtuales a museos, eventos históricos, diseños arquitectónicos o simular problemas humanos o técnicos como en procedimientos quirúrgicos, manejo o creación de maquinarias, entre otros. Por lo que, debido a su sencillez de captar la atención del alumnado, permite relacionarse con diferentes contenidos de cualquier materia (Gutiérrez et al., 2019). Además, elimina el costo de comprar y actualizar equipos en las aulas o laboratorios, reduce al mínimo los riesgos de seguridad y disminuye el uso de objetos peligrosos o muy valiosos, como también los gastos de viajes (Heidari, 2020).

Por lo que, ante los usos educativos, se deberían de analizar varios aspectos que intervienen en el aprendizaje del estudiante, pero se está dando importancia a la creatividad, siendo su concepto la generación de ideas o productos novedosos y útiles. Así mismo la creatividad tiene algunos factores como la motivación y estado de flujo que debido a la inmersión de la RV (Yang et al., 2018), genera una buena actitud que sería la motivación, que a su vez contribuye a la concentración a la tarea dada definida como estado de flujo, que nos llevaría a la creatividad del alumno.

En cuanto a la motivación, se define como la buena disposición de los estudiantes a participar en actividades de clase y sus razones para hacerlo. Además, la motivación se compone de la interacción de cuatro dimensiones: atención, relevancia, confianza y satisfacción. (Gutiérrez et al., 2019). 
Por otro lado, estudios previos han demostrado que cuando los individuos entran en estado de flujo tienden a mostrar un mayor rendimiento creativo. Siendo su concepto de una situación psicológica altamente positiva y agradable que ocurre cuando las personas apenas pueden notar sus actividades y a veces ni siquiera se dan cuenta de sí mismas. (Yanget al., 2018). Teniendo la teoría o estado de flujo las siguientes dimensiones: concentración, sentido de control, pérdida de la noción del tiempo y pérdida de la autoconciencia. (Gonçalves, 2018). Es así que sin esta condición de la creatividad no fluirían las ideas con facilidad.

De este modo, se aborda esta la temática, ya que los estudiantes requieren el desarrollo de actividades educativas a través de la creatividad las que pueden ser desarrolladas, por medio de la motivación y del estado de flujo. El objetivo del artículo es analizar como la realidad virtual mejora la experiencia educativa en pregrado. Considerando que la realidad virtual mejora la creatividad a través de la motivación y el estado de flujo, mejorando las habilidades para el aprendizaje en los estudiantes de pregrado.

\section{DESARROLLO}

En el aprendizaje en la enseñanza de pregrado, se requiere que los estudiantes obtengan hábitos mentales cognitivos y creativos, mediante el uso de aplicativos de RV, así mismo, se requiera que los profesores hagan la búsqueda y selección de programas cognitivos, para que cuando les den el debido valor a dichas aplicaciones educativas, den su salto de fe y los integren a las clases con estrategias de hábitos mentales (Steele et al., 2019). Por lo que es importante que los docentes estén siempre actualizados en el uso de las TICs y sepan cómo y qué sofware de RV puede contribuir en sus asignaturas.

De esta manera, las realidades virtuales tienen la facilidad de integrar a los estudiantes en entornos novedosos y motivadores, así como propiciar que se concentren solo en la tarea o problema. Además, permite relacionar a los estudiantes a situaciones únicas y manipulables para obtener resultados creativos. Siendo aparentemente las limitaciones de la RV sobrecargar cognitivamente a los alumnos con información o sensaciones no necesarias, disminuyendo la productividad cognitiva (Grigorenko, 2019). Por lo que se entiende que los programas a utilizar no deben de tener escenarios o procedimientos distractores que dificulten el aprendizaje del alumno.

Así mismo, se sabe que los alumnos que reciben retroalimentación o comentarios recordatorios en un entorno de RV, logran llegar mejor al rendimiento creativo. De esta forma el mecanismo de retroalimentación o recordatorio no solo ayuda a los participantes a que se concentren, sino que también los insta a pensar más sobre nuevas ideas (Yan et al., 2019). Asumiendo de esta manera, que las clases de RV, deben de estar acompañadas con una metodología de aprendizaje, reiterando lo aprendido para propiciar el resultado cognitivo y creativo que requieren los estudiantes.

También, la RV es una plataforma para soluciones experimentales, que, al no causar ninguna consecuencia negativa real, permite a los estudiantes, tener menos presión, logrando así buscar y encontrar resultados creativos a los problemas (Heidari, 2020), por lo que al lograr la RV, el acceso a diversos escenarios difíciles de conseguir en forma real, motiva a 
los alumnos a no sentir temor de experimentar. De esta manera, hacen que las operaciones del entorno de realidad virtual sean fáciles de usar, mejorando sus niveles de aprendizaje, los que pueden resultar en un mayor enfoque para el proceso creativo (Yang et al., 2018). Siendo así que, por su virtualidad, la RV contribuye a que mejoren varios aspectos de la creatividad como el estado de flujo.

Como ya se mencionó, el estado de flujo es promovido por la realidad virtual en las clases de pregrado, además es adquirido por medio de retos lúdicos que puedan cumplir los alumnos, quienes realizan actividades con RV, parecen estar en estado de flujo, ya que se encuentran completamente inmersos en un sentimiento de enfoque con plena participación y disfrute en el proceso de la actividad. Asimismo, se aprecia que pueden responder correctamente las preguntas de desafío de equipo. Por lo que también se vincula el estado de flujo con la construcción de camaradería y la interacción con sus participantes (Punako, 2018), ello se tiene que tomar en cuenta en los aprendizajes lúdicos en grupo.

También, el uso de la RV puede obtener un estado del flujo, ya que los usuarios se sienten más inmersivos, concentrados y creativos. Así se aclara que involucrarse en un mundo irreal que hace que la atención sea mayor lo que contribuye a asimilar mejor la información y por medio de la teoría de flujo ser más creativos (Gonçalves \& Camposo, 2018). Por lo que, se debe estar en un ambiente virtual, lo suficientemente inmersivo, para mantener la ilusión de estar en otro espacio y no perder la concentración, del mismo modo, el alumno debería de conservar la expectativa de lograr el objetivo propuesto por el docente. De esta manera, el uso de la RV da la sensación de estar en un escenario del mundo real, que influye positivamente en la inmersión, por lo que se sugiere fortalecer el sentimiento de encarnación y presencia (Kampling, 2018). Lo que se puede reforzar con accesorios además de los visores de RV, para que la interacción sensorial sea más completa.

Además, la experiencia de flujo se produce si el nivel de desafío no es ni demasiado alto ni demasiado bajo que el nivel de habilidad de, en este caso, el alumno. Por lo que, si no se proporciona este equilibrio, no puede experimentar apatía, ansiedad o relajación, debiendo permitir que se sientan seguros de su capacidad y control en su entorno de aprendizaje. (Bagriacik Yilmaz \& Banyard, 2020). Por lo que las actividades mediante la RV deben tener retos que se puedan cumplir para propiciar la fluidez en los aprendizajes y creatividad.

Igualmente, queda claro que la RV es fácil de usar y proporciona una mejor experiencia de flujo en la mayoría de los participantes, evidenciado debido a que los usuarios se muestran satisfechos con su trabajo y disfrutan de un estado de ánimo positivo y agradable al momento de crear. También la condición experimental inmersiva de la RV, contribuyó al rendimiento, el flujo, la atención y la meditación de la creatividad del producto de los individuos. Del mismo modo los estudiantes que confiaron en las señales visuales y la información contextual logran aumentar más su rendimiento de resolución de problemas. (Yan et al., 2019). Por lo que se entiende el estar concentrado y disfrutar del uso de la RV genera mayor fluidez creativa.

Asimismo, se conoce que el estado de flujo está relacionado con la satisfacción de los universitarios en el uso de la RV en laboratorios, también, elimina los límites de tiempo y espacio, mejorando el rendimiento de los estudiantes, como las de habilidades de indagación, 
habilidades prácticas, percepción y habilidades analíticas. Por lo que se recomienda crear un escenario de alta interacción entre los alumnos y el entorno virtual, para fortalecer su experiencia de flujo, siendo fundamental algunos mecanismos de desafío como la gamificación, que podrían ayudar a aumentar la participación de los estudiantes (Zhang et al., 2020). Por lo que es recomendable las actividades de aprendizaje lúdicos mediante los juegos entre estudiantes.

Al teorizar el estado de flujo, como uno de los objetivos importantes en los entornos de $\mathrm{RV}$, se entiende que se debe mejorar la motivación en los estudiantes, mediante el uso de juegos. Además, se sabe que la teoría de flujo funciona independientemente de la calidad tecnológica y que está influenciada por la voluntad, el estado de ánimo y la disposición de los usuarios (Shin, 2018). De esta manera una parte importante para lograr el desarrollo del estado de flujo es la motivación, la cual es originada por la novedad del uso de programas nuevos (Campos Soto et al., 2020), por lo que se considera que la RV por su estado de inmersión mejora la motivación al promover la atención, el interés y la curiosidad de los alumnos durante las clases. En tal sentido, la motivación debe contar con elementos físicos y virtuales suficientes a fin de que cada estudiante regule su autoaprendizaje. También son necesarias las indicaciones del profesor para alcanzar los logros de las competencias de sus clases, las que deben ser preparadas previamente para que los alumnos puedan resolver problemas, de tal forma que el aprendizaje incluya funciones cerebrales como el razonamiento, la memoria y la atención. Lo que permitirá el aprovechamiento de la RV y la participación activa de los estudiantes a través de la argumentación, la proposición y la resolución (Tovio et al., 2019). Por lo que es necesario acompañar a esta herramienta de aprendizaje una adecuada metodología junto con el seguimiento del docente. Cabe precisar que la inclusión de RV en la experiencia educativa, no garantiza el interés, la motivación o la comprensión del contenido, a menos que estos recursos se utilicen en un entorno educativo adecuado (Altomari, 2017); siendo importante la selección adecuada de programas de RV, la calidad de inmersión y la metodología de aprendizaje.

También, es importante para lograr la motivación de los estudiantes, que primero el docente sea el motivado usando la RV, a fin de que luego motive a sus alumnos. Debiendo ser presentado como una herramienta de aprendizaje lúdico que incrementa el interés y la motivación de los estudiantes, la cual dependerá de qué tan nueva sea la herramienta virtual para que se sienten más motivados (Díaz-López et al., 2019) debido a la expectativa de uso (Özgen et al., 2019), quedando claro que mientras más novedosa sea la interacción del alumno con al RV, mayor será su interés y la atención.

De esta forma la RV proporciona una experiencia de aprendizaje inmersiva que ayuda a los alumnos a explorar el contenido educativo e interactuar con lo virtual, minimizando las distracciones internas y externas, lo que promueve el conocimiento, retención y aumenta la motivación de los estudiantes. Además, la RV hace factible el aprendizaje personalizado controlando el proceso, como estudiar a su propio ritmo, practicar según sea necesario, y reflexionar más (Heidari, 2020).

En síntesis, la RV es una herramienta tecnológica con un gran potencial para estimular positivamente la motivación y la interactividad en el aprendizaje, en especial la atención que surge si el alumno advierte una brecha entre su conocimiento actual y el que se está adqui- 
riendo involucrándolos en una experiencia única e inolvidable. De esta manera, facilita una motivación con una inmersión emocional que mantenga la atención y el interés, despertando la curiosidad generando sensaciones creadas a partir de estímulos viso-auditivos, que de otro modo serían imposibles de alcanzar (Gutiérrez et al., 2019), constituyendo un aporte importante el uso RV al asociarla con ambientes inusuales en el que con una metodología adecuada estarían lo suficientemente motivados para el aprendizaje.

\section{CONCLUSIONES}

Luego de un análisis se concluye que la RV mejora la creatividad a través de la motivación y el estado de flujo, desarrollando habilidades para el aprendizaje en los estudiantes de pregrado, fomentando su uso y la adaptación de los programas existentes a las clases, para que los alumnos generen más soluciones creativas en los retos y problemas existentes.

Asimismo, se evidencia que el estado de flujo es promovido por la RV en las clases de pregrado por medio de retos lúdicos que puedan cumplir los alumnos, por lo que es importante que se implementen tareas en forma de juegos colaborativos, y así logren explorar, recopilar, compartir e integrar el conocimiento durante el proceso de generación de ideas creativas.

Por último, la RV promueve la motivación en las clases de pregrado a través de la atención y curiosidad, siendo importante que los sistemas sean más inmersivos, mediante el uso de equipos propios de las universidades y la capacitación de los docentes, por lo que se requerirá que se planifique un presupuesto para la implementación y uso de esta tecnología.

\section{REFERENCIAS}

Altomari, A. G. P. (2017). Realidad virtual y realidad aumentada en la educación, una instantánea nacional e internacional. Economía creativa, (7), 34-65.

Bagriacik Yilmaz, A., \& Banyard, P. (2020). Engagement in Distance Education Settings: A Trend Analysis. Turkish Online Journal of Distance Education, 21(1), 101-120.

Campos Soto, M. N., Navas-Parejo, M. R., \& Moreno Guerrero, A. J. (2020). Realidad virtual y motivación en el contexto educativo: Estudio bibliométrico de los últimos veinte años de Scopus. ALTERIDAD. Revista de Educación, 15(1), 47-60.

Díaz-López, L., Ortiz, J. T., \& Contreras, C. P. (2019). Strategies for inclusive and safe education using virtual reality: from the digital library perspective. Digital Library Perspectives. https://www.emerald.com/insight/content/doi/10.1108/DLP-08-20190034/full/html

Gómez Sánchez, M. (2018). Test de usabilidad en entornos de Realidad Virtual. No Solo Usabilidad, (17). http://www.nosolousabilidad.com/articulos/test_usabilidad_ realidad_virtual.htm

Gonçalves, F., \& Campos, P. (2018). Mild place illusion: a virtual reality factor to spark creativity in writing. In Proceedings of the 36th European Conference on Cognitive Ergonomics (pp. 1-8). https://dl.acm.org/doi/10.1145/3232078.3232085 
Grigorenko, E. L. (2019). Creativity in Digital Reality/Creatividad en la realidad digital. Studies in Psychology, 40(3), 585-607.

Gutiérrez, R. C., Somoza, J. A. G. C., Taranilla, R. V., \& Armero, J. M. M. (2019). Análisis de la motivación ante el uso de la realidad virtual en la enseñanza de la historia en futuros maestros. Edutec. Revista Electrónica De Tecnología Educativa, (68), 1-14.

Heidari, F. (2020). Introduce Virtual Reality to College Technical Training ProgramIntensified VR Training for Safety and Economic Efficiency. American Society for Engineering Education. https://peer.asee.org/34871.pdf

Kampling, H. (2018, January). The role of immersive virtual reality in individual learning. In Proceedings of the 51st Hawaii International Conference on System Sciences. https://scholarspace.manoa.hawaii.edu/bitstream/10125/50060/paper0173.pdf

Özgen, D. S., Afacan, Y., \& Sürer, E. (2019). Usability of virtual reality for basic design education: a comparative study with paper-based design. International Journal of Technology and Design Education, 1-21. http://repository.bilkent.edu.tr/bitstream/ handle/11693/53030/Usability_of_virtual_reality_for_basic_design_education_a_ comparative_study_with_paper_based_design.pdf? sequence=1

Punako, R. (2018). Computer-Supported Collaborative Learning using Augmented ana Virtual Reality in Museum Education [Doctoral Dissertation, College of Engineering and Computing Nova Southeastern University]. https://nsuworks.nova.edu/cgi/ viewcontent.cgi?article $=2041 \&$ context $=$ gscis_etd $/$

Shin, D. (2018). Empathy and embodied experience in virtual environment: To what extent can virtual reality stimulate empathy and embodied experience? Computers in Human Behavior, 78, 64-73.

Steele, P., Johnston, E., Lawlor, A., Smith, C., \& Lamppa, S. (2019). Arts-Based instructional and curricular strategies for working with virtual educational applications. Journal of Educational Technology Systems, 47(3), 411-432. https://journals.sagepub.com/ doi/abs/10.1177/0047239518803286

Tomalá Suárez, P. J., \& Obando Chonillo, B. S. (2018). Tecnologías educativas en el desarrollo del pensamiento creativo [Bachelor's thesis, Universidad de Guayaquil, Facultad de Filosofía, Letras y Ciencias de la Educación].

Tovio, D. J. M. T., Barraza, E. A. J. B., Mangones, D. A. P., \& Guerra, I. D. V. (2019). Uso de la realidad virtual en entornos educativos para la enseñanza y el aprendizaje de los procesos quirúrgicos y asépticos. http://clei2019.utp.ac.pa/storage/app/uploads/ public/5d8/d00/43e/5d8d0043e125d750584799.pdf

Yang, X., Lin, L., Cheng, P. Y., Yang, X., \& Ren, Y. (2019). Which EEG feedback works better for creativity performance in immersive virtual reality: The reminder or encouraging feedback? Computers in Human Behavior, 99, 345-351.

Yang, X., Lin, L., Cheng, P. Y., Yang, X., Ren, Y., \& Huang, Y. M. (2018). Examining creativity through a virtual reality support system. Educational Technology Research and Development, 66(5), 1231-1254.Zhang, M. H., Su, C. Y., Li, Y., \& Li, Y. Y. (2020). Factors affecting Chinese university students' intention to continue using virtual and remote labs. Australasian Journal of Educational Technology, 36(2), 169-185. 